\title{
Visual Cues supporting Direct Touch Gesture Interaction with In-Vehicle Information Systems
}

\author{
Ronald Ecker ${ }^{1}$ \\ ${ }^{1}$ BMW Group Research and Technology \\ Hanauerstraße 46 \\ Munich, Germany \\ Ronald.Ecker@bmw.de
}

\author{
Katja Hertzschuch ${ }^{1}$ \\ Andreas Butz ${ }^{2}$ \\ ${ }^{2}$ University of Munich \\ Media Informatics \\ Amalienstraße 17 \\ Munich, Germany
}

\begin{abstract}
Recent in-vehicle information systems are increasingly equipped with touch screens. While classic (i.e. point-based) direct touch interaction has known benefits in non-automotive environments, it primarily relies on visual attention, which makes it a bad candidate for interaction in the car, where visual attention should be on the road. We have designed an interaction scheme for IVIS based on touch gestures and pie menus and implemented several versions of it featuring visual cues as improvements to the original idea. In an extensive user study with a primary driving task, we were able to show that our interaction scheme is significantly faster than classic touch interaction and that it demands shorter gesture using visual cues.
\end{abstract}

\section{Categories and Subject Descriptors}

H.5.2 [Information Interfaces and Presentation]: User Interfaces Evaluation/methodology, Input devices and strategies, Interaction styles; I.3.6 [Computer Graphics] Methodology and Techniques Interaction techniques.

\section{General Terms}

Performance, Design, Experimentation, Human Factors.

\section{Keywords}

Automotive HCI, touch screens, touch gestures, in-vehicle information system (IVIS), pie menu, automotive user studies.

\section{INTRODUCTION}

Current car cockpits are providing many tertiary functions such as navigation, entertainment and communication via integrated invehicle information systems (IVIS). One common possibility to interact with IVIS is to use a touch screen.

In general interaction approaches based on touch-sensitive displays are a promising possibility to handle the complexity of infotainment systems in an intuitive manner - assuming that users can spend their full visual attention on the interaction. In mobile environments, especially in a car, users have only limited resources for the interaction task itself. The driver's focus must be

Copyright held by author(s)

AutomotiveUI'10, November 11-12, 2010, Pittsburgh, Pennsylvania ACM 978-1-4503-0437-5 on the primary task - in our case maneuvering a car safely. Interacting with the IVIS has a lower priority [1]. This means that beside general usability criteria for user interfaces, IVIS have to fulfill special suitability criteria to ensure traffic safety. Usability requirements such as learnability, efficiency, memorability, error handling and satisfaction are crucial for user acceptance [2]. On the other hand, avoiding driver distraction, interruptability and reducing visual attention are essential preconditions for usage in a car. ISO standards [3][4] and negotiated agreements[1][5] provide detailed requirements for IVIS, e.g., for total task times and glance behavior while interacting.

Visual attention is almost as valuable a resource while interacting with a touch-sensitive display as during the primary tasks of driving. The driving task can be divided into three levels: stabilization, maneuvering and navigation [6]. During stabilization, drivers have to keep the car within the lanes and keep their distance to other road users. Hence, they must observe their environment and react to traffic participants' behavior. Maneuvering categorizes tasks like turning and overtaking. The task of navigating involves knowledge about the route.

For touch screen interaction with an IVIS, users have to examine the display in order to detect visual objects they want to select. Furthermore the finger must be coordinated to the visual display object without any tactile lead. For this procedure, visual attention is indispensable. In addition, ergonomic drawbacks for touch screen interaction with IVIS include the missing haptic feedback as a reaction to a users input. The placement of the display inside the cockpit must be a trade-off between readability and reachability of the display. However, touch-based interaction also offers many advantages, e.g., its intuitiveness and efficiency compared to interaction approaches based on tangible controllers. Furthermore touch interaction is not limited to tapping touch buttons anymore. Using touch gestures or multi-touch technology opens new possibilities, especially for mobile interaction with reduced visual attention.

[17] showed the potential of touch gestures for interacting with an IVIS via a pie menu based interface called pieTouch (see Figure 1). In this study we want to go one step further and answer questions about glance behavior of touch gesture based IVIS in comparison to point-touch interaction. The second issue is the investigation of using visual cues to enhance the usability and learnability of touch gestures and pie menus in IVIS. We developed one basic pie menu prototype with touch gestures and a corresponding system with point-touch input. Additionally we realized alternative touch gesture based prototypes with varying visual highlighting mechanisms of the target menu entry. A 80 comparison of all systems in a standardized dual task evaluation 
method called Lane Change Task (LCT) showed no differences in glance behavior between touch gestures and point-touch, but enhancements in efficiency and user acceptance, when using visual cues.

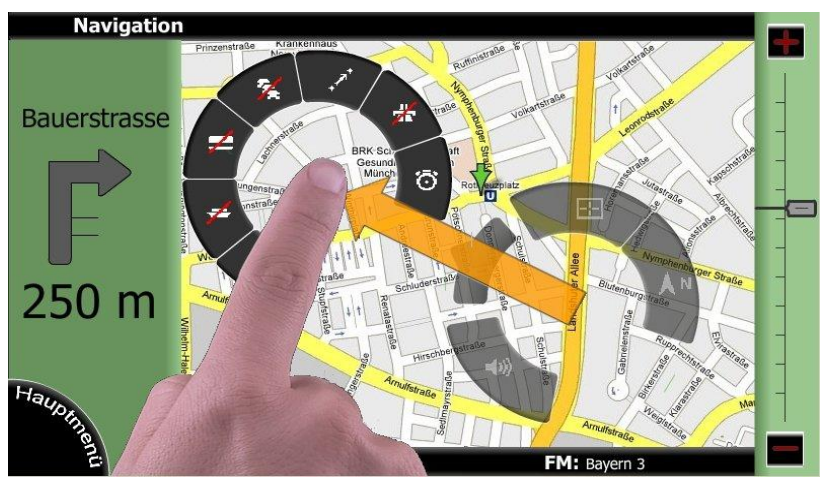

Figure 1: Pie menu for navigation functions in the map. The menu appears after laying down the finger on the screen. An option is selected by dragging into the direction of the target menu entry and executed by lifting up the finger.

\section{RELATED WORK}

The most popular example of touch gestures is Apples iPhone. Besides tapping the screen for menu and function selection, pinch and spread gestures can be used to enlarge or to minimize pictures or web pages. Lists are scrolled upwards, downwards or sideway by using drag gestures. A flick - which means the horizontal movement of the finger for example in a photo album - is used to look at the next picture [12][13]. In this way, operating becomes quite easy, position-independent and therefore enables an almost blind interaction.

Even for devices used in a non-critical main task, such as mobile phones, menus affording little visual attention are a worthwhile improvement. Pie menus, which arrange menu items in circular order around an initial point show potential in blind interaction. Selecting menu item takes less time and reduced visual attention in comparison to list-based menus, if the properties have been chosen in the right way [7]. Opening a pie menu can be triggered by different actions like a mouse click or touching the screen. By dragging the finger or mouse into the direction of the desired menu item the corresponding function is selected. The selection is executed by lifting the finger. Therefore the selection can be corrected before lifting the finger. According to Kurtenbach [19] the number of menu items should be 4,8 or 12 , where smaller numbers decrease the seeking time, enlarge the surface of menu entries and increase the performance. Due to a better symmetry the velocity of use increases, if the number of items is even, because the arrangement, for example a clock or compass, is well known. To speed up the process of learning, inverse terms such as open and close have to be arranged on opposite sides, in order for users to remember them quicker. Hopkins [20] advises a menu with a maximum of 8 items to ensure that users can distinguish the directions easily.

The wave menu [21] applies the pie menu to the user interface of a mobile phone. To guarantee that the submenus are always displayed on the screen, the root menu appears around the cursor. To select an item, the user draws a mark in the desired direction.

Brandl et al [22] demonstrate in their work a concept employing a multi-touch table. When a user is interacting with his finger, parts of the screen - especially of the menu - can be covered by the hand. To eliminate this disadvantage, the menu provides a gap in the position which can be obscured. Depending on the hand direction, the system rearranges the menu to prevent occlusion. In addition, the inner section of the pie menu is reserved for gestures.

The earPod [25] implements a pie menu in which single items are read out by moving the finger above the elements. Whenever the border of an item is crossed, a clicking sound can be heard. If the user pauses his finger on the item, the label will be read out until he moves further. The study shows that the earPod can be operated without a secondary task a little slower handled more quickly. Moreover, participants were able to memorize the directions of the offered functions, so they were able to select a particular item directly without looking.

Pirhonen introduces a music player [15], which can be controlled blind by using gestures. He achieved significantly faster task times by conducting a dual task evaluation, namely absolving a slalom course during interaction. Auditory feedback informs the participant about the current state and the accuracy of the gesture. A simple tap starts or stops the player. Dragging the finger up or down on the screen controls the volume. By dragging to the right or left the next or previous song is selected.

After market navigation systems are mainly controlled via touch screens. All producers use a grid-based hierarchical menu. The handling of these devices is not optimized for the use whilst driving, which is therefore not recommended by the manufacturer. The reason for this is the increased visual distraction due to the hand-eye-coordination and an ergonomically bad position.

More and more car manufacturers integrate touch screens into their vehicles for handling the IVIS. Mostly a hybrid solution of touch interaction and traditional haptic user interfaces is realized. This overcomes the disadvantage of the high visual attention needed by list- or grid-based touch.

Audi A8 presented the new multimedia interface, which combines its conventional centrally mounted multifunctional controller with a touch pad. To simplify the input of a new navigation target, letters and numbers can be entered with gestures. The navigation map can be shifted by a short drag of the finger [17].

MyFord will be integrated in the Ford Edge and Lincoln MKX from the beginning of 2011, and its functions will be controlled by a touch-screen. The system can be adapted to the customer's needs. Various objects of the interface can be tapped. Furthermore gestures can be used for certain functions such as cover flow [26].

Also the design study Up! from Volkswagen should allow drivers to interact with the air condition, navigation and music system by direct touch gestures [23].

Bach [16] compared the use of a standard car radio with a pointtouch- and a gesture-based interface. A driving experiment, implemented with a speed of $50-70 \mathrm{~km} / \mathrm{h}$ shows differences in lateral errors, which implicates a loss of control over the vehicle, caused by increased attention to the user interface. Users made significantly more errors using the normal radio, than when using the others. The point touch system could be handled quicker. However only 19 percent of total glances could be assigned to the gesture based system, 39 to the point touch and 42 to the normal radio. The gesture-based system was most popular with participants and was rated the most intuitive, quickest and easiest to use. The point-touch-based system was ranked in second place, whereas the ordinary radio was challenged due to its small buttons and poor layout. This study points out that direct touch gestures can reduce visual distraction and improve the driver's 
Several studies have investigated the conditions under which graphical objects, such as buttons or list items improve touch interaction in static environments. The size of a finger has to be considered, for instance. The diameter of an adult finger can vary between 16 and 22 millimeters, and in general older people have wider [24] fingers. General references for the size of an object vary between 10 and 22 millimeters [28]. For automotive environments, no uniform standards are known.

\section{PROBLEM STATEMENT}

[17] presented a new approach for interacting with IVIS via direct touch gestures called pieTouch. It combines touch gestures with a visual circular menu, which appears around the touch point, when the user is tapping the screen. The functionality and represented menu options are designed as a pie menu.

By using a LCT, they compared the gesture-based pieTouch system with an equivalent point-touch-based system. The results showed that the gesture-based pie menu reduces total task times, shows a favorable learning curve and increases user acceptance.

As visual attention is a critical factor for interacting with IVIS we want to investigate in the following, whether it is possible to reduce the visual demand of a gesture-based touch approach using pie menus during driving. We assume that users have to be trained very well to achieve a reduction of visual attention, even if they are conducting a higher priority task at the same time. Because of this, we try to increase efficiency and speed up learning processes by using visual cues for selecting an item of a pie menu.

\subsection{Glance Behavior}

During the comparative study between the point-touch-based system and pieTouch, the investigator determined an approximately blind use of the main pie menu in the domain of navigation after the third task repetition during the experiment. No eye tracking system was used in [17] to record the glance behavior of the participants. As a result there is a lack of objective data to verify the investigator's observation.

In a point-based touch system, users need at least one glance to identify and hit the button for opening the menu and an additional glance for selecting the desired menu entry in a second interaction step. It always entails two steps requiring visual attention independent of the learning level. In comparison, in a gesturebased pie menu system, users do not necessarily need a glance towards the screen. Assuming that the menu invocation is not position-independent as in [17], for the first step of detecting the button and touching it one glance is needed. Afterwards, users can drag the finger in the direction of the required menu entry without looking at the display again, if they know the menu by heart. This assumption is verified in diverse user studies regarding pie menu interaction in other domains like desktop computing [7].

In this study, we compare a point-touch menu with a corresponding pie menu during the lane change task. Additionally we record the user's glance behavior with the DIKABLIS eye tracking system. As a result we are able to compare glance behavior of the two systems and verify if highly trained users need less visual attention with the touch-gesture-based system. Furthermore we investigated if the systems fulfill automotive requirements. [1] specifies that $85 \%$ of the glances have to be under a threshold of 1.5 seconds.

\subsection{Visual Cues}

Our approach to increase the suitability and usability of pie menus in automotive environments is to apply different kinds of visual cues. In the moment the user is dragging the finger into a direction, the targeted menu item will be highlighted. Thereby we suppose that the user is able to recognize faster, which item is selected and when the system reacts to his movements. In this report we want to compare different types of visual cues (size, color and rubber band) to verify this assumption and to identify the approach with the most potential concerning efficiency, learnability, visual distraction and user acceptance.

Fitts`s law states that the duration for reaching an object depends on its distance and on its size. Hence size is one factor which is processed in preattentive selection. User studies have shown that task completion times and error rates can be reduced by enlarging the target item [8]. Based on these theories and studies, the target pie menu element will be expanded by approximating it.

Color is another crucial parameter in preattentive perception and can be used to highlight an object from its environment. Search time can be reduced [9][10] and we use color as another promising parameter for highlighting an entry of our pie menu. In an informal pretest we evaluated that one highlighting color is more useful than using different colors for different menu entries.

Inspired by the game world of goo [11], we connect the touch point of the user's finger and menu entry by a thin line, which adapts dynamically to finger movements like a rubber band. This alternative combines coloring the desired menu entry with an explicit reference via the rubber band.

The second focus of our user study beside glance behavior of point touch versus touch gestures, compares these alternatives amongst each other and with a version without visual cues during the LCT.

\section{SYSTEM DESIGN}

To answer our questions about glance behavior during interaction with touch gestures and if visual cues can enhance usability in IVIS, we implemented several prototypes. To generate a realistic procedure and to give the participants an idea of a future use case, a map is displayed, on which three Points of Interest (POI), a gas station, a restaurant and a hotel are arranged. The task is to find the gas station and to open a pie menu by touching it. Afterwards users can select a certain menu item, by dragging their finger in the direction of the target. The function is executed by lifting the finger from the screen. All three POIs are randomly located after each menu selection.

The pie menu consists of eight circular menu items with a diameter of 1.2 centimeters. The start circle has an active area with a diameter of two centimeters, of which only a smaller circle with a diameter of 1.2 centimeters is visible (iceberg tip) for the user. The distance between start and target circles measures 2.4 centimeters. These parameters are determined based on previously executed user studies. We used eight simple symbols as menu entries in order to simulate an unknown menu (moon, plus, square, triangle, circle, heard, minus and star).

As long as the finger is touching the screen, there is a bright circle displayed around it, to show the reaction of the system to the finger. An acoustic signal rings out, after the finger is released from the screen, to confirm the menu item. Figure 2 shows the five implemented menu alternatives with and without visual cues. In the following the alternatives are explained.

\subsection{Size}

Once the finger moves into the direction of a menu item, the diameter increases from 1.2 to 1.95 centimeters. As a result, lower 
task times and lower error rates should be achieved [8]. Figure 2a shows the behavior of the system.

\subsection{Color}

For the Color prototype a colored ring appears around the target menu item. For this, just one color is used, see Figure 2b.

\subsection{Rubber Band}

In the third alternative a visible connecting line between finger and target menu item appears (see Figure 2c). Inspired by the computer game World of Goo [11], the line will be adapted dynamically to the finger's movement like a rubber band. The link connects the finger directly to the menu item and highlights it.

\subsection{Base}

For investigating the effect of the implemented visual support, one prototype without any visual highlighting was realized (see Figure 2d).

\subsection{Point-Touch}

For the purpose of comparing the use of gestures in an unknown menu with a conventional point touch system, there is a fifth menu variety. After touching the POI icon, the menu opens and stays standalone unclosed, to enable tapping on the target menu item directly. The menu items of this system are arranged exactly as in the gesture interfaces. This was done in order to vary only one variable, the interaction technique (see Figure 2e). The items have an invisible enlargement (iceberg), thus the activation area of the item has a diameter of two centimeters.

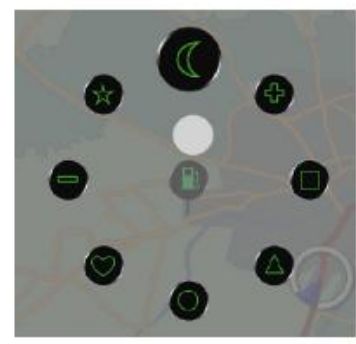

(a) V1-Size

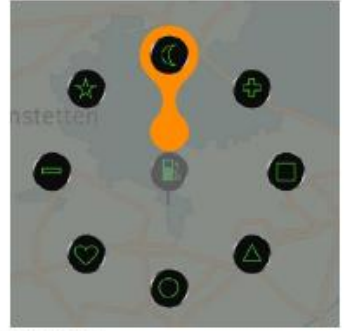

(c) V3-Rubber

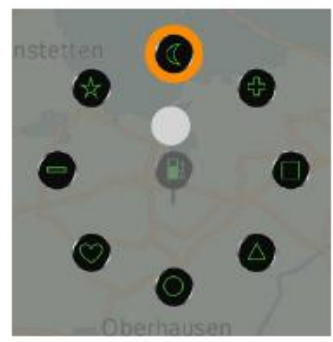

(b) V2-Colar

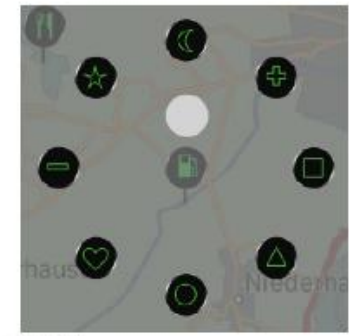

(d) V4-Base

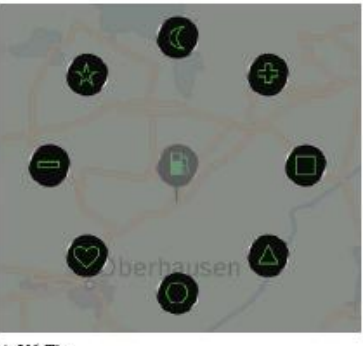

(e) V6-Tip
Figure 2: The five kinds of menus.

\section{USER STUDY}

In order to evaluate automotive systems, dual task experiments are usually conducted. In order to test the developed visual cues supporting direct touch gestures we applied a method for laboratory investigations (LCT) combined with an eye tracking system (DIKABLIS) capturing user gaze behavior. The point touch system and the gesture-based pie menu system without any visual highlighting are the control conditions. This should allow drawing conclusions about the differences between point-touch and touch gestures in terms of total task time (TTT) and gaze behavior.

\subsection{Lane Change Task (LCT)}

The LCT is a driving simulation in which participants perform certain lane changes indicated by designated traffic signs. Simultaneously participants have to interact with the IVIS. As a result, the mean lane deviation (Figure 3 ) from the ideal driving line provides evidence about the visual and cognitive distraction from the primary task of driving while using the system to be tested. The LCT imitates driving and its characteristics. The traffic signs at the side of the simulated road indicate the lane onto which the participant should change immediately. Participants are instructed to give priority the main goal of the LCT, namely changing the lane as fast as possible and keeping a constant speed of $60 \mathrm{~km} / \mathrm{h}$ - which in this case stands for full throttle. The simulation is controlled via a Logitech steering wheel, a break and an accelerator. The driving scene is displayed on a 17', display according to the ISO standard.

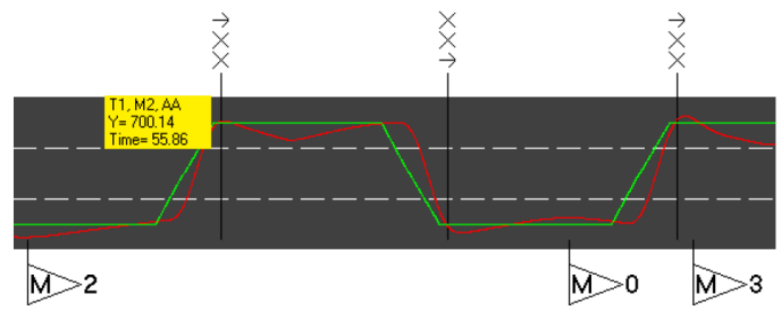

Figure 3: Results of the LCT (green line = ideal driving line, red line $=$ driven line $)$.

To analyze the driving data, the LCT analysis tool returns the total area between the normative model and the actual driving course (m2) divided by distance ( $m$, mean lane deviation, MDEV) and a visual plot of the ideal and the driven driving line (3).

\subsection{Eye Tracking System}

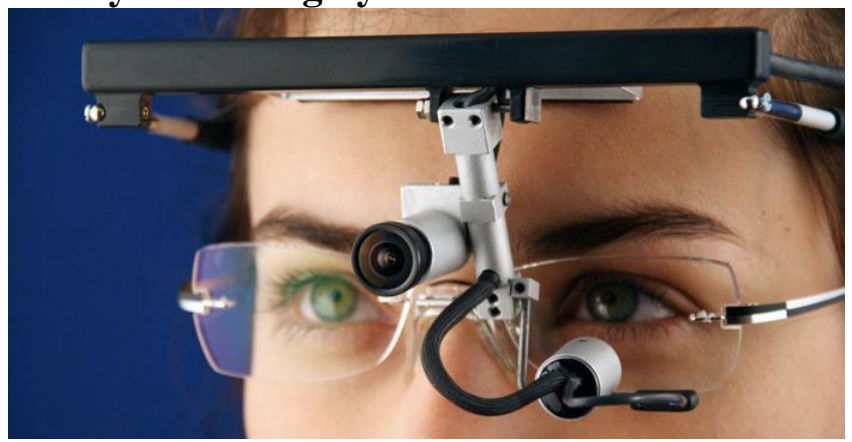

Figure 4: Dikablis eye tracking system.

With the eye tracking system Dikablis visual distraction during interacting with an in-car system can be determined. Therefore participants had to wear glasses with two cameras, one for 
recording the pupil movements of the left eye and another camera that records the scene (Figure 4).

Before starting the evaluation, both cameras must be calibrated using a special tool in order to ensure the compliance of the eye and scene movie. For an automatic data analysis, visual markers must be assembled around the areas of interest. With the D-Lab tool these areas of interest (AOI) can be defined and considered relative to the visual markers. Different variables such as the number of gazes, mean gaze duration, minimum and maximum gaze duration can be computed for each AOI.

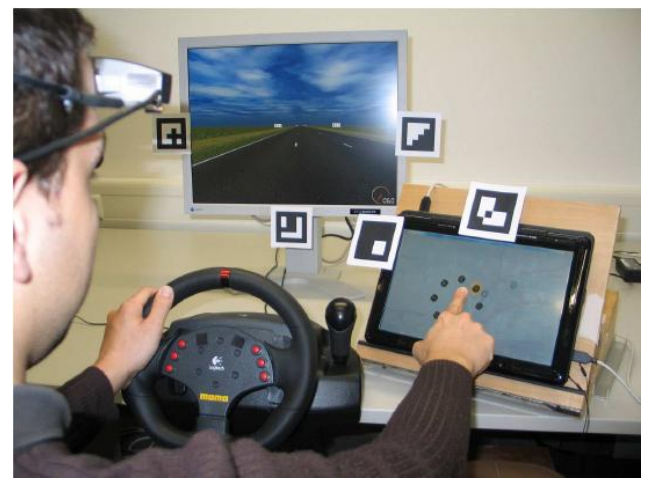

Figure 5: Experimental setup for the LCT containing visual markers for Dikablis.

Figure 5 illustrates the experimental setup. The touch screen for the systems was located in a realistic position combining ideal readability and interaction during driving. To avoid fatigue of the users arm during the execution of gestures an arm rest was placed in front of the touch screen.

\subsection{Participants}

28 volunteers aged between 22 and 59 years attended. The average age was 37 years. 23 male and five female persons took part in the experiment. 25 of the volunteers were right handed and three left handed. 26 (92\%) participants are adept in interaction with touch sensitive surfaces.

\subsection{Experimental Design}

All different visual cues in terms of prototypes were evaluated conducting a within subject design. Order was counterbalanced to avoid learning effects. The dependent variables are the mean glance time, the $80^{\text {th }}$ percentile of the maximum glance time, error rate, gesture length and the total task time. Independent variables are the different prototypes and the control conditions in terms of the point touch system and the one without visual cues. The names of the systems are: Size for the prototype using the size as visual help, Color of the one with color highlighting, Rubber Band the rubber band prototype, Base and Point-Touch for the control conditions.

The evaluation started with a demographic questionnaire followed by a questionnaire designed to gain insight into the experience of the volunteers in interacting with touch devices. Afterwards the prototypes are explained to the participants and trained until they felt secure in interacting. After this, the baseline condition of the driving simulation was driven. To ensure the familiarity of the participants with the LCT, a mean lane deviation of less than one meter must be achieved by the test person. In order to avoid predictable distances of the traffic signs indicating the lane change, nine different driving routes were randomly driven. Afterwards the interaction task was executed without driving simulation containing three times the selection of each circular aligned menu item for every single prototype ( 24 selection tasks, 8 menu items, 5 prototypes). After this training period, the same tasks had to be executed whilst driving. During this glance behavior was recorded with DIKABLIS. The instructions were not coordinated with the occurrence of the traffic signs. Hence users had to decide if the situation for choosing a menu item is appropriate. For invoking the pie menu, one of three randomly located POIs - the gas station - had to be touched. After every trial a questionnaire capturing the subjective user preferences were filled out.

\subsection{Hypotheses}

According to related studies and previous work following hypotheses should be tested:

H1: The TTT of a menu item selection by gestures is shorter when visual cues are used.

$\mathrm{H} 2$ : The selection of a menu item by gestures affords less visual attention when using visual cues.

H3: The gesture length is shorter when visual cues are supplementing the gesture interaction.

H4: Gesture length for the visual cues Rubber Band and Color is shorter than for Size.

H5: Gesture interaction reduces the visual distraction in comparison to point-touch.

H6: The TTT of the gesture interface is shorter than the TTT of the point-touch interface.

\subsection{Objective Results}

The dependent objective variable driving performance in terms of the mean lane deviation was captured by the LCT simulation, the gaze behavior by the eye tracking system DIKABLIS and the gesture length and total task time (TTT) by the prototypes. The error rate was recorded by the investigator.

\subsubsection{Total Task Time}

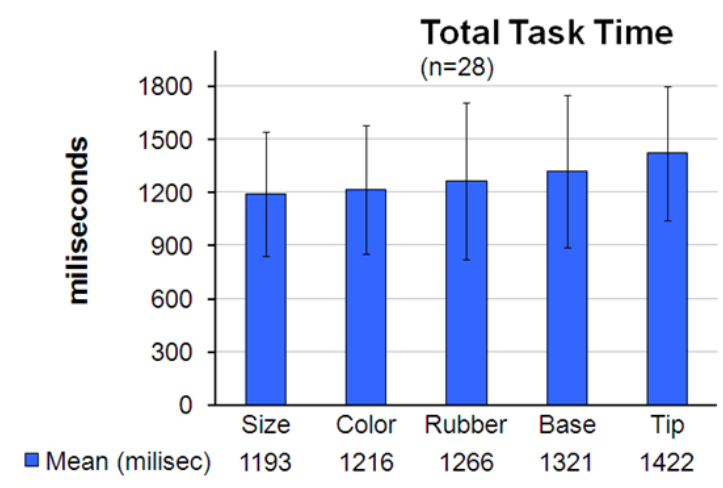

\begin{tabular}{|c|c|c|c|c|c|}
\hline Sig. & Size & Color & Rubber & Base & Tip \\
\hline Size & & & & & \\
\hline Color & 0,838 & & & & \\
\hline Rubber & 0,189 & 0,511 & & & \\
\hline Base & 0,001 & 0,001 & 0,003 & & \\
\hline Tip & 0,304 & 0,126 & 0,626 & 0,135 & \\
\hline
\end{tabular}

Figure 6: Total task time.

The TTT comprises the time from the first contact of the users' finger to the selection of the menu item and was measured in milliseconds. As the TTT was not normally distributed the 84 Friedman test was employed. This test shows that there are 
significant differences $\left(\mathrm{Chi}^{2}(5)=21.06, \mathrm{p}=.001\right)$ between the prototypes. The Wilcoxon test reveals that the Base prototype had a longer TTT than the Size $(\mathrm{p}<.001)$, Color $(\mathrm{p}<.001)$ and the Rubber Band $(p<.05)$ prototype (see Figure 6$)$. For the PointTouch prototype no significant differences to the other systems could be found $(\mathrm{p}=.135)$ although the descriptive data shows the highest TTT (1422 ms) within this prototype. Therefore H1 can be accepted and H6 must be rejected.

\subsubsection{Error Rate}

In total every volunteer had to choose 144 menu items. Therefore the proper POI for invoking the menu had to be touched. In the total experiment 33 mistakes of selecting the wrong POI occurred. For the overall error rate this means that 0.82 percent of these 4032 tasks were executed incorrectly. Another mistake participants made was that they lifted the finger after invoking the menu but without choosing a menu item. This happened 78 times (1.93 percent). 58 (1.44 percent) participants selected a wrong menu item. The Friedman test showed no significant differences $\left(\mathrm{Chi}^{2}(5)=7,63, \mathrm{p}=.178\right)$ between the different versions.

\subsubsection{Gesture Length}

In our case gesture length is defined by the distance between the start and end point of a gesture. After the correcting the degrees of freedom using the Greenhouse-Geisser correction $\left(\mathrm{F}_{4.42,} 2964\right.$ $\left.{ }_{23}=83.73, \mathrm{p}<.001\right)$ a significant impact of the prototypes could be found. The Base prototype was used with significantly longer gestures than all the other prototypes supporting the user with visual feedback (see Figure 7). H3 can be accepted whereas H4 must be denied because no significant differences could be found within the visual alternatives.

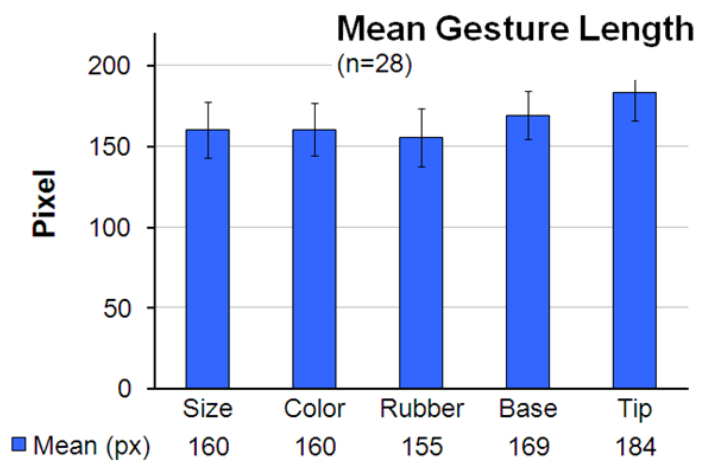

\begin{tabular}{|c|c|c|c|c|c|}
\hline Sig. & Size & Color & Rubber & Base & Tip \\
\hline Size & & & & & \\
\hline Color & 1,000 & & & & \\
\hline Rubber & 0,190 & 0,143 & & & \\
\hline Base & 0,000 & 0,000 & 0,000 & & \\
\hline Tip & 0,000 & 0,000 & 0,000 & 0,000 & \\
\hline
\end{tabular}

Figure 7: Gesture length in pixel (50px is one $\mathbf{c m})$.

\subsubsection{Driving Performance}

In terms of the mean lane deviation no significant difference could be found. On average the MDEV for the Size $1.20 \mathrm{~m}, 1.16 \mathrm{~m}$ for Color, $1.19 \mathrm{~m}$ for Rubber Band, $1.28 \mathrm{~m}$ for Base and $1.18 \mathrm{~m}$ for the point-touch prototype.

\subsubsection{Gaze Behavior}

The gaze behavior of the prototypes was manually separated by the investigator and started with the first instruction for the first menu item and ended with the selection of the last menu item.
The different visual cues and control conditions do not show a significant effect on total glance time (Figure 8) onto the touch screen $\left(\mathrm{F}_{2.86,71.59}=1.42, \mathrm{p}=.245\right)$.

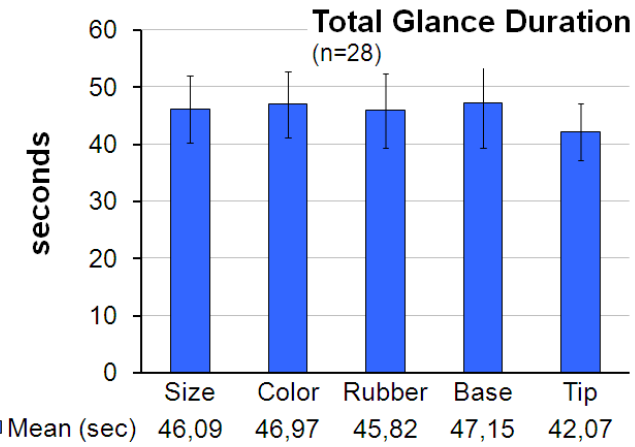

Figure 8: Total glance duration onto the touch screen.

The mean glance time of all prototypes is between 1.15 and 1.32 seconds. No significant impact of the prototypes could be found. The $80^{\text {th }}$ percentile of the maximum glance times which eliminates 20 percent of the uppermost outliers lies between 1.55 and 1.79 seconds. The Size prototype had the highest glance times (1.76 seconds) and the Point-Touch prototype the shortest (1.55 seconds) but no significant differences could be shown (Figure 9).

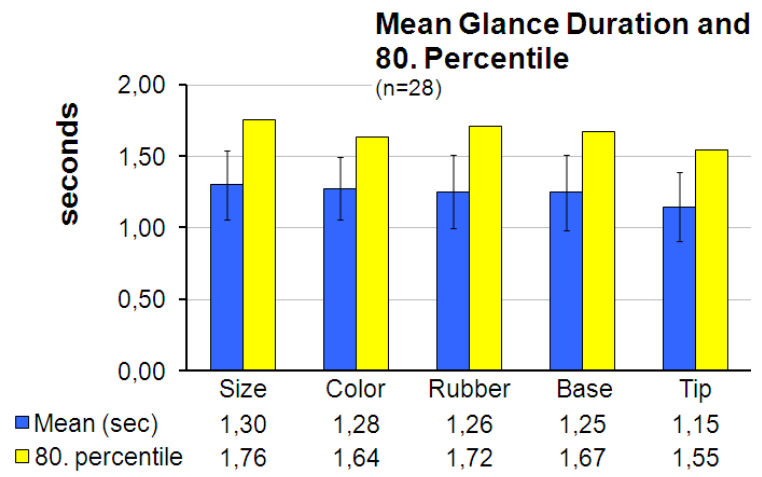

Figure 9: Mean glance duration and $80^{\text {th }}$ percentile.

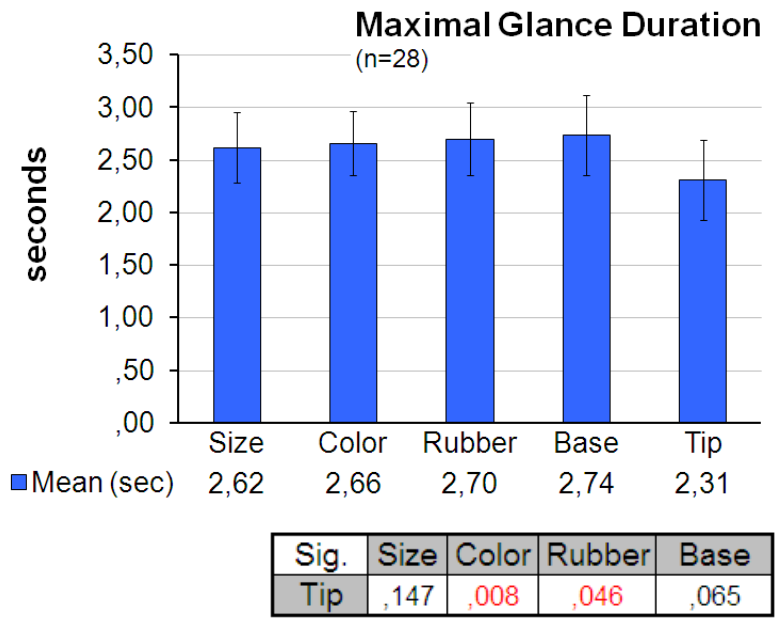

Figure 10: Maximal glance duration onto the touch screen.

Prototypes showed a significant impact on maximum glance time $\left(\mathrm{F}_{5}, \quad 125=3.208, \mathrm{p}=0.009\right)$. Pairwise comparisons revealed 
and the Color (2.66 seconds) could be found $(\mathrm{p}=.008)$. Also the difference between Point-Touch and Rubber Band $(\mathrm{p}=.046)$ are visible (Figure 10). Consequently $\mathrm{H} 2$ and $\mathrm{H} 5$ must be denied.

\subsection{Subjective User Preferences}

For retrieving the participants' subjective user opinion about the implemented versions of visual feedback one questionnaire and a comparative statement were used. The questionnaire intended to capture the user reaction immediately after interacting with each prototype and driving. The comparatives question had to be answered at the end of the experiment in order to get the favored visual feedback.

The questionnaire and the comparative statements are based on a nine-item Likert scale where 1 stands for I totally deny and 9 for $I$ fully agree.

The questionnaire for getting feedback about the user reaction after each trial comprises four statements which should be stated by the participants:

1. I was sure to select the correct menu entry in every situation. For this statement no significant impact could be found by the Friedman test. In general the rating was quite high (between 7.64 for Size and 8.00 for Point-Touch)

2. The selection of the menu entry was alleviated.

A significant impact of the different onto the users rating could be found $\left(\mathrm{F}_{3.54,95.56}=6.392, \mathrm{p}<.001\right)$. Pairwise comparisons revealed significant differences between Color (5.71) and Base (4.21) with $\mathrm{p}<.001$. Furthermore this statement was rated significantly higher for Rubber Band (5.86) than for Base $(\mathrm{p}<.05)$. Also the PointTouch (6.00) was rated higher than Base ( $\mathrm{p}<.05)$.

\section{I felt unpleasant during selecting a menu item.}

A significant main effect of the different variations could be found (F3.11, 83.98=5.253, p=.002). Rubber Band was judged significantly lower (3.75) than Base (5.14). In general the mean values of the rating were: $\mathrm{Size}=3.89$, Color $=3.86$, Rubber Band $=3.75$, Base $=5.14$, Point - Touch $=4.04$.

4. I felt save during driving and selecting a menu item.

No significant impact of the prototypes could be shown. The user rating was as follows: $\operatorname{Size}=5.07$, Color $=5.18$, Rubber $\mathrm{Band}=4.89$, Base $=4.36$, Point - Touch $=5.50$.

The comparative question at the end of the study was: Which visual feedback do you prefer? Eleven participants favored the Color, ten the Rubber Band, five the Size and two no visual feedback.

The responses regarding the favored interaction technique in which users had to decide if they prefer gestures or point-touch were astonishing. Twenty-three participants preferred the pointtouch and only 5 the gesture interface. This disagrees with previous results like Ecker et al [17].

\section{CONCLUSION}

In this work, we went one step further in investigating touch gestures for IVIS. The comparison of the different visual cues Size, Color, Rubber Band and the condition Base and Point-Touch reveals interesting evidences for the use of visual cues in automotive environments. On the one hand total task times could be significantly reduced by the visual feedback. All three variations were used faster than the gesture interface without any visual help. Furthermore the TTT produced by the point-touch prototype are the longest even though no significant differences could be found. Based on the captured error rate the gestures were used in similar accuracy than the point-touch. Consequently the shorter task time does not result in more errors. Also the gesture length could be reduced by implementing visual support for the user. The menu selection for Size, Color and Rubber Band was executed by shorter gesture than for Base. This result allows the assumption that users felt more secure with visual feedback which is supported by the subjective user opinion in question number 1 , 2 and 3 . The total glance duration, mean glance duration and $80^{\text {th }}$ percentile does not reveal any significant differences. For the maximum glance duration significant differences between the gesture interfaces Rubber Band and color and Point-Touch prototype could be found. Even the Base system shows tendencies $(\mathrm{p}=.065)$ to a higher maximum glance time than point-touch. Therefore we assume that the visual feedback is not the reason for the longer glances onto the touch screen which corresponds with the subjective user opinion.

In general participants favored the Color before the Rubber Band and Size. No visual feedback was rated the most undesirable. Very surprising was that 23 participants judged the point-touch prototype as the most suitable for driving. One reason for this result can be that the occlusion of menu items during interaction was not taken into account in our systems. This does not occur with the point-touch prototype. Moreover the lack of training and the chosen unknown and abstract menu could have let to the situation that participants had to recall the menu anew in every trail. Hence no blind and eye free operation could be executed. With the point-touch system the menu stays visible without touching the screen.

In summary, we found out that in unknown menus there is no difference in glance behavior between point and gesture touch pie menus. But in the end, we were able to demonstrate that visual cues can enhance usability of touch gestures in IVIS.

\section{ACKNOWLEDGMENTS}

Our thanks to Tessa Hannemann for carefully proof reading this work and all participants of the user study.

\section{REFERENCES}

[1] Driver Focus-Telematics Working Group, Alliance of Automobile Manufacturers. 2003 Statement of Principles, Criteria and Verification Procedures on Driver Interaction with Advanced In-Vehicle Information and Communication Systems

[2] Nielsen, Jakob. 1993 Usability Engineering. Acad. Press, 1993. - 8-10, 49-70 S. - ISBN 0-12-518405-0

[3] DIN Deutsches Institut für Normung (eds.). 2003 Road vehicles - Ergonomic aspects of transport information and control systems - Specifications and compliance procedures for in-vehicle visual presentation. DIN Deutsches Institut für Normung, Oct. 2003. - Ref.No.: DIN EN ISO 15008:200310.

[4] DIN Deutsches Institut für Normung (eds.). 2003 Road vehicles - Ergonomic aspects of transport information and control systems - Dialogue management principles and compliance procedures. DIN Deutsches Institut für Normung, Mar. 2003. - Ref.No.: DIN EN ISO 15005:2003.

[5] European Commission Information Society and Media Directorate-General - G4 ICT for Transport (eds.). 2005 European Statement of Principles on the Design of Human Machine Interaction (ESoP 2005). 
[6] Bernotat, R. 1970 Plenary Session: Operation Function in Vehicle Control. In Anthropotechnik in der Fahrzeugführung, Applied Ergonomics, Vol 13

[7] Callahan, J., Hopkins D., Weiser M., Shneiderman B. An empirical comparison of pie vs. linear menus. In CHI '88: Proceedings of the SIGCHI conference on Human factors in computing systems, pages 95-100, New York, NY, USA, 1988. ACM.

[8] M. Fitts, Paul. The information capacity of the human motor system in controlling the amplitude of movement. 1954. Journal of Experimental Psychology, 121(3):262-269, September.

[9] E. Christ, R. Review and analysis of color coding research for visual displays (in aircraft). Human Factors, 17(6):542570, 121975.

[10] D. L. Fisher and K. C. Tan. Visual displays: the highlighting paradox. Hum. Factors, 31(1):17-30, 1989.

[11] 2D Boy. World of goo. <http://2dboy.com/games.php>, 21.03. 2010.

[12] Apple. iphone, ipad, magic mouse. <http://www.apple.com/de/>, 08.03. 2010.

[13] Ralph Wagner. 10 gründe für den iphone-erfolg. <http://gestaltungenaffoltern.wordpress.com/2009/09/28/10grunde-fur-den-iphone-erfolg/>, 08.03. 2010.

[14] Ralf Sander and DPA. Der PC im Couchtisch. $<$ http://www.stern.de/digital/computer/microsoft-surface-derpc-im-couchtisch-590113.html>, 08.03.2010.

[15] Antti Pirhonen, Stephen Brewster, and Christopher Holguin. Gestural and audio metaphors as a means of control for mobile devices. In CHI '02: Proceedings of the SIGCHI conference on Human factors in computing systems, pages 291-298, New York, NY, USA, 2002.ACM.

[16] Kenneth Majlund Bach, Mads Gregers Jaeger, Mikael B. Skov, and Nils Gram Thomassen.You can touch, but you can't look: interacting with in-vehicle systems. In CHI '08: Proceeding of the twenty-sixth annual SIGCHI conference on Human factors in computing systems, pages 1139-1148, New York, NY, USA, 2008. ACM.

[17] Ecker, R., Broy, V., Butz, A., De Luca, A. pieTouch: A Direct Touch Gesture Interface for Interacting with InVehicle Information Systems. In 11th International Conference on Human-Computer Interaction with Mobile Devices and Services (MobileHCI 2009), Bonn, Germany, September 15 - 18, 2009.

[18] Audi. A8 - Ausstattung - Multi Media Interface. $<$ http://www.audi.de/de/brand/de/neuwagen/a8/a8/design/int erieur.html\#source=http://www.audi.de/de/brand/de/neuwage n/a8/a8/ausstattung/multi_media_interface/mmi_touch.html \&container=page $>, 07.03 .2010$.

[19] Gordon Paul Kurtenbach. The design and evaluation of marking menus. PhD thesis, Toronto,Ont., Canada, Canada, 1993.

[20] Don Hopkins. The design and implementation of pie menus. Dr. Dobb's J., 16(12):16-26, 1991.

[21] Jeremie Francone, Gilles Bailly, Laurence Nigay, and Eric Lecolinet. Wavelet menus: a stacking metaphor for adapting marking menus to mobile devices. In MobileHCI '09: Proceedings of the 11th International Conference on HumanComputer Interaction with Mobile Devices and Services, pages 1-4, New York, NY, USA, 2009. ACM.

[22] Peter Brandl, Jakob Leitner, Thomas Seifried, Michael Haller, Bernard Doray, and Paul To. Occlusion-aware menu design for digital tabletops. In CHI EA '09: Proceedings of the $27^{\text {th }}$ international conference extended abstracts on Human factors in computing systems, pages 3223-3228, New York, NY, USA, 2009. ACM.

[23] Auto Scout 24. Studie: VW Up! Lite. Noch ein Up-leger. $<$ http://ww2.autoscout24.de/magazine/mz_home/mz_home?a rticle $=149392>, 07.03 .2010$.

[24] Dan Saffer. Designing Gestural Interfaces: Touch screens and Interactive Devices. O'Reilly Media, Inc., 2008.

[25] Shengdong Zhao, Pierre Dragicevic, Mark Chignell, Ravin Balakrishnan, and Patrick Baudisch. Earpod: eyes-free menu selection using touch input and reactive audio feedback. In CHI '07: Proceedings of the SIGCHI conference on Human factors in computing systems, pages 1395-1404, New York, NY, USA, 2007. ACM.

[26] The Ford Story. Myford Defines a new Driver Experience. $<$ http://www.thefordstory.com/smarttechnology/myford\%E2\%84\%A2-defines-a-new-driverexperience/>, 17.01.2010.

[27] Pekka Parhi, Amy K. Karlson, and Benjamin B. Bederson. Target size study for one-handed thumb use on small touch screen devices. In MobileHCI '06: Proceedings of the 8th conference on Human-computer interaction with mobile devices and services, pages 203-210, New York, NY, USA, 2006. ACM.

[28] Seungyon Lee and Shumin Zhai. The performance of touch screen soft buttons. In CHI '09: Proceedings of the 27th international conference on Human factors in computing systems, pages 309-318, New York, NY, USA, 2009. AC 